\title{
Energy-Efficient Dual-Iteration Power Allocation for Two-Phase Relay System with Massive Antennas
}

\author{
Long Zhao, ${ }^{1}$ Wei Xiang, ${ }^{2}$ Jie Mei, ${ }^{1}$ Hui Zhao, ${ }^{1}$ Hang Long, ${ }^{1}$ and Lin Li ${ }^{1}$ \\ ${ }^{1}$ Wireless Signal Processing \& Network Lab, Key Laboratory of Universal Wireless Communication, Ministry of Education, \\ Beijing University of Posts and Telecommunications (BUPT), Beijing 100876, China \\ ${ }^{2}$ School of Mechanical and Electrical Engineering, University of Southern Queensland, Toowoomba, QLD 4350, Australia
}

Correspondence should be addressed to Long Zhao; z.long.gm@gmail.com

Received 17 January 2014; Accepted 16 April 2014; Published 5 May 2014

Academic Editor: Periklis Chatzimisios

Copyright ( 92014 Long Zhao et al. This is an open access article distributed under the Creative Commons Attribution License, which permits unrestricted use, distribution, and reproduction in any medium, provided the original work is properly cited.

\begin{abstract}
This paper considers the scenario where multiple source nodes communicate with multiple destination nodes simultaneously with the aid of an amplify-and-forward relay equipped with massive antennas. In order to achieve optimal energy efficiency (EE) of the entire relay system, this paper investigates the power allocation problem for the multiple pairs of nodes at both the source nodes and the relay node, where the relay employs the backward and forward zero-forcing filters. Since the EE optimization problem cannot be solved analytically, we propose a two-phase power allocation method. Given power allocation of one phase, the optimal power allocation is derived for the other phase. Furthermore, two dual-iteration power allocation (DIPA) algorithms with performance approaching that of optimal EE are developed based on the instantaneous and statistic channel state information, respectively. Numerical results show that the proposed DIPA algorithms can greatly improve EE while guaranteeing spectrum efficiency (SE) when compared with the equal power allocation algorithm. Moreover, both algorithms suggest that deploying a rational number of antennas at the relay node and multiplexing a reasonable number of node pairs can improve on the EE and SE.
\end{abstract}

\section{Introduction}

With the rapid development of smart terminals and their new applications, the traffic of wireless communication networks grows exponentially [1]. Moreover, power consumption caused by the networks results in excessive carbon emissions and unaffordable operator expenditure [2]. Therefore, both spectrum efficiency (SE) and energy efficiency (EE) are considered as significant metrics for evaluating the performance of communication systems. In recent years, massive multiple-input multiple-output (MIMO) has been put forward to simultaneously serve tens of users when the base station (BS) employs hundreds of antennas [3]. Massive MIMO can not only improve SE, but also enhance system EE $[3,4]$. Apart from these advantages, massive MIMO is able to enhance the link reliability due to its higher diversity gains [5], reduce interuser interference, and guarantee physical layer security owing to the extremely narrow beam $[5,6]$. In a massive MIMO system, individual antenna failure of the antenna array will not be catastrophic to the system performance $[5,6]$. The scheduling scheme can be made simply due to the channel harden phenomenon [7]. Certainly, since the maximum number of pilot sequences is bounded by the coherent time and bandwidth, pilot contamination due to pilot reuse in multicell scenarios imposes the ultimate limit on the performance of massive MIMO system $[3,5,6]$.

Meanwhile, maximum ratio transmission (MRT)/zero forcing (ZF) and maximum ratio combination (MRC)/ZF techniques have been proven as practical precoders and detectors $[5,6]$. The performance, including SE, EE, and reliability, of massive MIMO with different precoders/detectors has been investigated broadly for single cell or multicell [4, $8,9]$. However, relays with massive antennas serving multiple pairs of nodes are less studied. Such a relay system is a type of isolated system, which exchanges less necessary or no information with the networks. Taking the stricken area as an example, a temporary relay can be deployed, which is mainly used for mutual communication by rescue workers or local 
residents. As depicted in Figure 1, the ergodic SE of multiple pairs of nodes served by an amplify-and-forward (AF) relay with the ZF/MRT filter was analyzed when the number of antennas at the relay goes to infinity [10]. However, realtime SE with a finite number of antennas is more significant for a practical relay system. Moreover, realistic EE should be evaluated in consideration of both radiated and circuit powers.

This paper investigates real-time power allocation at both source nodes and relay node with a finite number of antennas in order to achieve optimal EE for the entire relay system. When the two-phase AF relay employs the backward and forward ZF filters, both the real-time SE and the lower bound of ergodic SE are first analyzed. Then, the EE optimization problem is formulated based on a realistic power consumption model, which consists of both radiated and circuit powers. Due to the intractable objective function, the optimization problem is decomposed into a two-phase power allocation problem. The optimal single-phase power allocation is derived when the power allocation of the other phase is given using convex optimization theory. Furthermore, considering both the real-time SE and ergodic SE, two dual-iteration power allocation (DIPA) algorithms capable of achieving near optimal EE performance are developed for the entire relay system, which can simultaneously improve $\mathrm{EE}$ and SE compared with the equal power allocation (EPA) algorithm.

The remainder of this paper is organized as follows. Section 2 introduces the system model and analyzes the system SE. The EE optimization problem is formulated in consideration of both radiated and circuit powers in Section 3. The optimal single-phase power allocation is derived and two DIPA algorithms for the entire relay system are developed in Section 4. Section 5 presents simulation results and Section 6 presents the conclusion of this paper.

Notation. Uppercase boldface letters and lowercase boldface letters denote matrices and vectors, respectively. $(\cdot)^{\mathrm{T}},(\cdot)^{\mathrm{H}}$, and $(\cdot)^{-1}$ represent the transpose, conjugate transpose, and pseudoinverse of matrix/vector, respectively. $\operatorname{diag}\{\mathbf{a}\}$ is a diagonal square matrix whose main diagonal is formed by vector a. $\|\cdot\|$ represents the Euclidian 2-norm. Consider $[x]_{+}=\max \{x, 0\}$ and $[x]_{a}^{b}=\min \{\max \{a, x\}, b\} . \mathscr{E}[\cdot]$ represents mathematical expectation. $\mathcal{N}\left(\mu, \sigma^{2}\right)$ denotes the real Gaussian distribution with mean $\mu$ and variance $\sigma^{2}$. $\mathscr{C} \mathcal{N}\left(\mu, \sigma^{2}\right)$ is a complex Gaussian distribution with mean $\mu$ and real/imaginary component variance $\sigma^{2} / 2$.

\section{System Model and Spectrum Efficiency}

A two-phase AF relay system model is illustrated in Figure 1. Multiple single-antenna source nodes $\mathbf{S}=\left\{\mathrm{S}_{1}, \mathrm{~S}_{2}, \ldots, \mathrm{S}_{K}\right\}$ communicate with multiple single-antenna destination nodes $\mathbf{D}=\left\{\mathrm{D}_{1}, \mathrm{D}_{2}, \ldots, \mathrm{D}_{K}\right\}$ assisted by the relay node (R) equipped with $N$ antennas, and $S_{k} \rightarrow D_{k}$ is a pair of nodes. In order to simplify the simulation in Section 5, the source and destination nodes are distributed in an area with minimum radius $r_{0}$, maximum radius $R_{0}$, and angle $\theta$. But those

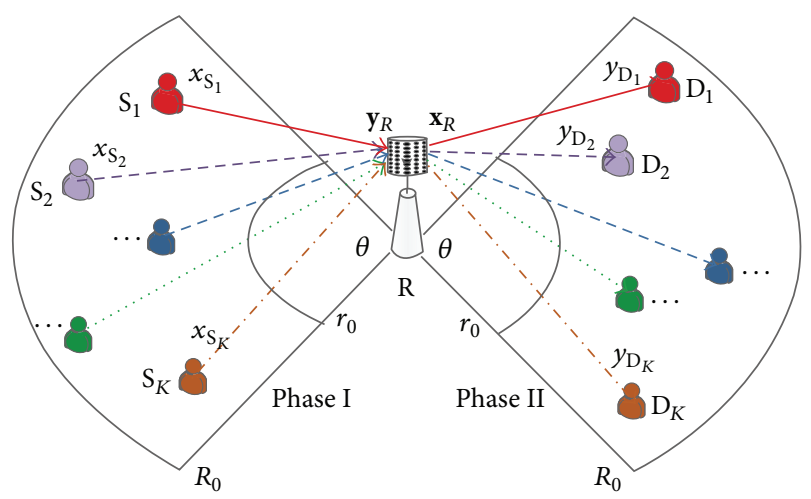

FIGURE 1: Relay system serving multiple pairs of nodes with massive antennas.

assumptions have no effect on the theoretical deduction. This paper considers block Rayleigh fading channels and assumes that the perfect channel state information (CSI) can be acquired at $\mathrm{R}$. We also assume that the timely power allocation information can be transmitted to nodes $\mathbf{S}$ or $\mathbf{D}$ free of errors after R implementing the power allocation algorithm.

In phase I, $\mathbf{S}$ transmits signals to $\mathrm{R}$, and the received signals can be expressed as

$$
\mathbf{y}_{\mathrm{R}}=\mathbf{G}_{\mathrm{SR}}\left(\operatorname{diag}\left\{\mathbf{p}_{\mathrm{S}}\right\}\right)^{1 / 2} \mathbf{x}_{\mathrm{S}}+\mathbf{n}_{\mathrm{R}},
$$

where $\mathbf{x}_{\mathrm{S}}=\left[x_{\mathrm{S}_{1}}, x_{\mathrm{S}_{2}}, \ldots, x_{\mathrm{S}_{K}}\right]^{\mathrm{T}}$ is the modulated transmission symbol vector and $\mathrm{E}\left[\mathbf{x}_{\mathrm{S}} \mathbf{x}_{\mathrm{S}}^{\mathrm{H}}\right]=\mathbf{I}_{K} \cdot \mathbf{p}_{\mathrm{S}}=\left[p_{\mathrm{S}_{1}}, p_{\mathrm{S}_{2}}, \ldots, p_{\mathrm{S}_{K}}\right]^{\mathrm{T}}$ is the power allocation vector at $\mathbf{S}$. The channel matrix from $\mathbf{S}$ to $\mathrm{R}$ is $\mathbf{G}_{\mathrm{SR}}=\mathbf{H}_{\mathrm{SR}} \mathbf{D}_{\mathrm{SR}}^{1 / 2}$. The large-scale fading matrix $\mathbf{D}_{\mathrm{SR}}=$ $\operatorname{diag}\left\{\beta_{S_{1} R}, \beta_{S_{2} R}, \ldots, \beta_{S_{K} R}\right\}$ and $\beta_{S_{k} R}=\phi d_{S_{k} R}^{-\alpha} \xi_{S_{k} R}$, where $\phi$ is a constant related to the carrier frequency and antenna gain, $d_{\mathrm{S}_{k} \mathrm{R}}$ is the distance between $S_{k}$ and $\mathrm{R}, \alpha \in[2,6]$ is the path loss exponent, and $\xi_{S_{k} \mathrm{R}}$ is a log-normal shadow fading variable with distribution $10 \log _{10} \xi_{\mathrm{S}_{k} \mathrm{R}} \sim \mathscr{C} \mathscr{N}\left(0, \sigma_{\mathrm{sh}, \mathrm{S}_{k} \mathrm{R}}^{2}\right)$. The elements of the fast fading matrix $\mathbf{H}_{\mathrm{SR}}=\left[h_{n, k}\right] \in \mathscr{C}^{N \times K}$ are independent and identically distributed (i.i.d.) random variables with the distribution of $\mathscr{C} \mathscr{N}(0,1) . \mathbf{n}_{\mathrm{R}} \sim \mathscr{C} \mathcal{N}\left(0, \sigma_{\mathrm{R}}^{2} \mathbf{I}_{N \times N}\right)$ is the additive white Gaussian noise (AWGN) at the relay node $\mathrm{R}$.

At R, a backward ZF filter $\mathbf{B}=\left[\mathbf{b}_{1}^{\mathrm{T}}, \mathbf{b}_{2}^{\mathrm{T}}, \ldots, \mathbf{b}_{K}^{\mathrm{T}}\right]^{\mathrm{T}}=$ $\left(\mathbf{H}_{\mathrm{SR}}^{\mathrm{H}} \mathbf{H}_{\mathrm{SR}}\right)^{-1} \mathbf{H}_{\mathrm{SR}}^{\mathrm{H}}$ is employed, and its normalization matrix is $\mathbf{Q}_{\mathrm{B}}=\left(\operatorname{diag}\left\{q_{\mathrm{B} 1}, q_{\mathrm{B} 2}, \ldots, q_{\mathrm{B} K}\right\}\right)^{-1 / 2}$, where $q_{\mathrm{B} k}=\beta_{\mathrm{S}_{\mathrm{k}} \mathrm{R}} p_{\mathrm{S}_{k}}+$ $\left\|\mathbf{b}_{k}\right\|^{2} \sigma_{\mathrm{R}}^{2}(k=1,2, \ldots, K)$. The signals after the backward filter can be written as

$$
\mathbf{x}_{\mathrm{R}}=\mathbf{Q}_{\mathrm{B}} \mathbf{B} \mathbf{y}_{\mathrm{R}} .
$$

In phase II, R transmits signal $\mathbf{x}_{R}$ to $\mathbf{D}$, and the received signals at $\mathbf{D}$ can be expressed as

$$
\mathbf{y}_{\mathrm{D}}=\mathbf{G}_{\mathrm{RD}} \mathbf{F Q}_{\mathrm{F}}\left(\operatorname{diag}\left\{\mathbf{p}_{\mathrm{R}}\right\}\right)^{1 / 2} \mathbf{x}_{\mathrm{R}}+\mathbf{n}_{\mathrm{D}},
$$

where $\mathbf{p}_{\mathrm{R}}=\left[p_{\mathrm{R}_{1}}, p_{\mathrm{R}_{2}}, \ldots, p_{\mathrm{R}_{K}}\right]^{\mathrm{T}}$ is the power allocation vector at $\mathbf{R}$ for the multiple pairs of nodes. The channel 


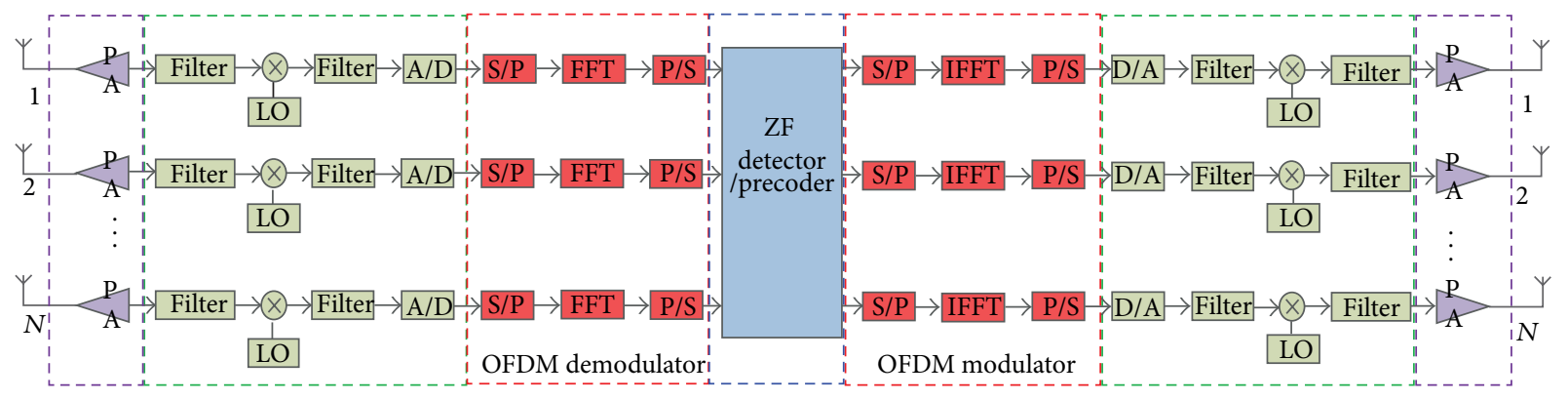

FIgURE 2: Components of power consumption at the relay node.

from $\mathrm{R}$ to $\mathbf{D}$ is $\mathbf{G}_{\mathrm{RD}}=\mathbf{D}_{\mathrm{RD}}^{1 / 2} \mathbf{H}_{\mathrm{RD}}$. The large-scale fading matrix $\mathrm{D}_{\mathrm{RD}}=\operatorname{diag}\left\{\beta_{\mathrm{RD}_{1}}, \beta_{\mathrm{RD}_{2}}, \ldots, \beta_{\mathrm{RD}_{K}}\right\}$ and $\beta_{\mathrm{RD}_{k}}=$ $\phi d_{\mathrm{RD}_{k}}^{-\alpha} \xi_{\mathrm{RD}_{k}}$, where $\phi$ and $\alpha$ are identical as in phase I, $d_{\mathrm{RD}_{k}}$ is the distance between $\mathrm{R}$ and $\mathrm{D}_{k}$, and $\xi_{\mathrm{RD}_{k}}$ is a lognormal shadow fading variable with the distribution of $10 \log _{10} \xi_{\mathrm{RD}_{k}} \sim \mathscr{C} \mathcal{N}\left(0, \sigma_{\mathrm{sh}, \mathrm{RD}_{k}}^{2}\right)$. The fast fading matrix $\mathbf{H}_{\mathrm{RD}}=$ $\left[h_{k n}\right] \in \mathscr{C}^{K \times N}$ contains i.i.d. $\mathscr{C} \mathcal{N}(0,1)$ entries. Consider $\mathbf{F}=$ $\left[\mathbf{f}_{1}, \mathbf{f}_{2}, \ldots, \mathbf{f}_{K}\right]=\mathbf{H}_{\mathrm{RD}}^{\mathrm{H}}\left(\mathbf{H}_{\mathrm{RD}} \mathbf{H}_{\mathrm{RD}}^{\mathrm{H}}\right)^{-1}$ is the forward ZF filter matrix at $\mathrm{R}$, and $\mathbf{Q}_{\mathrm{F}}=\left(\operatorname{diag}\left\{q_{\mathrm{F} 1}, q_{\mathrm{F} 2}, \ldots, q_{\mathrm{FK}}\right\}\right)^{-1 / 2}$ is its normalization matrix with $q_{\mathrm{F} k}=\left\|\mathbf{f}_{k}\right\|^{2} . \mathbf{n}_{\mathrm{D}}$ is the AWGN at D with the distribution of $\mathscr{C} \mathcal{N}\left(0\right.$, $\left.\operatorname{diag}\left\{\sigma_{\mathrm{D}_{1}}^{2}, \sigma_{\mathrm{D}_{2}}^{2}, \ldots, \sigma_{\mathrm{D}_{K}}^{2}\right\} \mathbf{I}_{K}\right)$.

The received signal-to-noise ratio (SNR) at $\mathrm{D}_{k}$ can be readily shown as

$$
\rho_{k}=\frac{a_{k} p_{\mathrm{S}_{k}} b_{k} p_{\mathrm{R}_{k}}}{a_{k} p_{\mathrm{S}_{k}}+b_{k} p_{\mathrm{R}_{k}}+1}
$$

where $a_{k}$ and $b_{k}$ are the channel-to-noise ratios (CNRs) of the links $\mathrm{S}_{k} \rightarrow \mathrm{R}$ and $\mathrm{R} \rightarrow \mathrm{D}_{k}$, respectively, and can be written as:

$$
\left[a_{k}, b_{k}\right]=\left[\frac{\beta_{S_{k} \mathrm{R}}}{\sigma_{\mathrm{R}}^{2}}\left\|\mathbf{b}_{k}\right\|^{-2}, \frac{\beta_{\mathrm{RD}_{k}}}{\sigma_{\mathrm{D}_{k}}^{2}}\left\|\mathbf{f}_{k}\right\|^{-2}\right]
$$

Furthermore, denoting the SE vector of multiple node pairs by $\mathbf{c}=\left[c_{1}, c_{2}, \ldots, c_{K}\right]^{\mathrm{T}}$, the overall SE of the entire relay system can be expressed as

$$
C_{\Sigma}=\sum_{k=1}^{K} c_{k}=\frac{1}{2} \sum_{k=1}^{K} \log _{2}\left(1+\rho_{k}\right)
$$

According to random matrix theory, both of the random variables $\left\|\mathbf{b}_{k}\right\|^{-2}$ and $\left\|\mathbf{f}_{k}\right\|^{-2}$ follow a gamma distribution with the parameters $(N-K+1,1)[11,12]$. Then, the expectations of random variables $\left\|\mathbf{b}_{k}\right\|^{2}$ and $\left\|\mathbf{f}_{k}\right\|^{2}$ can be expressed as $\mathscr{E}\left[\left\|\mathbf{b}_{k}\right\|^{2}\right]=\mathscr{E}\left[\left\|\mathbf{f}_{k}\right\|^{2}\right]=(N-K)^{-1}$. Owing to the convexity of function $\log _{2}(1+1 / x)$, the Jensen inequality $\mathscr{E}\left[\log _{2}(1+\right.$ $1 / x)] \geq \log _{2}(1+1 / \mathscr{E}[x])$ holds. Therefore, the lower bound of the SE of the relay system can be given as

$$
\bar{C}_{\Sigma}=\mathscr{E}\left[C_{\Sigma}\right] \geq \sum_{k=1}^{K} \bar{c}_{k}=\frac{1}{2} \sum_{k=1}^{K} \log _{2}\left(1+\bar{\rho}_{k}\right),
$$

where the equivalent SNRs and CNRs can be written as follows, respectively:

$$
\begin{gathered}
\bar{\rho}_{k}=\mathscr{E}^{-1}\left[\rho_{k}^{-1}\right]=\frac{\bar{a}_{k} p_{\mathrm{S}_{k}} \bar{b}_{k} p_{\mathrm{R}_{k}}}{\bar{a}_{k} p_{S_{k}}+\bar{b}_{k} p_{\mathrm{R}_{k}}+1}, \\
{\left[\bar{a}_{k}, \bar{b}_{k}\right]=\left[\frac{(N-K) \beta_{\mathrm{S}_{k} \mathrm{R}}}{\sigma_{\mathrm{R}}^{2}}, \frac{(N-K) \beta_{\mathrm{RD}_{k}}}{\sigma_{\mathrm{D}_{k}}^{2}}\right] .}
\end{gathered}
$$

The lower bound of ergodic SE in (7) is only dependent on the statistic CSI compared with the real-time SE in (6), which relates to the instantaneous CSI.

\section{Energy Efficiency Formulation}

According to [13], the power consumption model consists of both the radiated and circuit powers. The components of the source and destination nodes are similar to those in $[13,14]$. The components of power consumption for the relay node are depicted in Figure 2. Then, the power consumption models of the source, relay, and destination nodes are given as follows, respectively:

$$
\begin{aligned}
& P_{\mathrm{S \Sigma}}=\sum_{k=1}^{K}(\frac{\varsigma_{\mathrm{S}_{k}}}{\eta_{\mathrm{S}_{k}}} \underbrace{p_{\mathrm{S} k} B}_{\text {PA }} \underbrace{+p_{\mathrm{SC} 1} c_{k} B+}_{\text {coder\&modulator OFDM }} \underbrace{p_{\mathrm{SC} 2} B}_{\text {other }}+\underbrace{p_{1}}_{\mathrm{SC} 3}), \\
& P_{\mathrm{R} \Sigma}=\frac{\varsigma_{\mathrm{R}}}{\eta_{\mathrm{R}}} \sum_{k=1}^{K} \underbrace{p_{\mathrm{R} k} B}_{\mathrm{PA}}+\underbrace{p_{\mathrm{RC} 2} N B}_{\text {OFDM }}+\underbrace{p_{\mathrm{RC} 3} N}_{\text {filter,etc }}+\underbrace{p_{\mathrm{RC}}}_{\text {constant }} \\
& +\underbrace{p_{\mathrm{RC} 1}\left(7 N K^{2}+4 N K-2 K+7 K^{3}\right) B,}_{\text {ZF detector\&precoder }} \\
& P_{\mathrm{D} \Sigma}=\sum_{k=1}^{K}(\underbrace{p_{\mathrm{DC} 1} c_{k} B+}_{\text {demodulator\&decoder OFDM }} \underbrace{p_{\mathrm{DC} 2} B}_{\text {other }}+\underbrace{p_{0}}_{\mathrm{DC} 3}),
\end{aligned}
$$

where $\varsigma_{S_{k}}$ and $\varsigma_{R}$ represent the peak to average power ratios (PAPRs) of OFDM at the source nodes and relay node, respectively. $\eta_{\mathrm{S}_{k}}$ and $\eta_{\mathrm{R}}$ denote the efficiencies of the power amplifiers (PAs) at the source nodes and relay node, respectively. $B$ is the system bandwidth. $p_{\mathrm{SC} i}(i=1,2,3)$, $p_{\mathrm{RC} i}(i=1,2,3,4)$, and $p_{\mathrm{DC} i}(i=1,2,3)$ are the constant coefficients of circuit power consumption at the source, relay, and destination nodes, respectively. 
Therefore, the total power consumption per $\mathrm{Hz}$ of the whole relay system can be written as

$$
\begin{aligned}
P_{\Sigma} & =\frac{1}{B}\left(P_{\mathrm{S} \Sigma}+P_{\mathrm{R} \Sigma}+P_{\mathrm{D} \Sigma}\right) \\
& =\sum_{k=1}^{K}\left(\frac{\varsigma_{\mathrm{S}_{k}}}{\eta_{\mathrm{S}_{k}}} p_{\mathrm{S} k}+\frac{\varsigma_{\mathrm{R}}}{\eta_{\mathrm{R}}} p_{\mathrm{R} k}+p_{\mathrm{C} 1} c_{k}\right)+g(N, K, B),
\end{aligned}
$$

where $p_{\mathrm{C} 1}=p_{\mathrm{SC} 1}+p_{\mathrm{DC} 1}$ and

$$
\begin{aligned}
g(N, K, B)= & p_{\mathrm{RC} 1}\left(7 N K^{2}+4 N K-2 K+7 K^{3}\right) \\
& +p_{\mathrm{RC} 2} N+\left(p_{\mathrm{SC} 2}+p_{\mathrm{DC} 2}\right) K \\
& +\frac{1}{B}\left[p_{\mathrm{RC} 3} N+\left(p_{\mathrm{SC} 3}+p_{\mathrm{DC} 3}\right) K+p_{\mathrm{RC} 4}\right] .
\end{aligned}
$$

Based on the metric J/bit, the optimal EE problem of the overall relay system can be formulated as

$$
\begin{gathered}
E_{\mathrm{b}}^{\mathrm{o}}=\min E_{\mathrm{b}}=\min \left\{\frac{P_{\Sigma}}{C_{\Sigma}}\right\} \\
\text { s.t. }\left\{\begin{array}{l}
\mathrm{T}_{\mathrm{S}}: 0 \leq p_{\mathrm{S}_{k}} \leq P_{\mathrm{S}_{k}, \max }, \quad k=1,2, \ldots, K, \\
\mathrm{~T}_{\mathrm{R}}: 0 \leq \sum_{k=1}^{K} p_{\mathrm{R}_{k}} \leq P_{\mathrm{R}, \text { max }},
\end{array}\right.
\end{gathered}
$$

where $P_{\mathrm{S}_{k} \text {,max }}$ and $P_{\mathrm{R}, \max }$ are the maximum transmission powers at $S_{k}$ and $R$, respectively.

\section{Energy-Efficient Power Allocation}

4.1. DIPA Based on Instantaneous CSI. It is easy to see that (15) is a computationally intractable problem according to convex optimization theory. In this part, given the power allocation vector $\mathbf{p}_{\mathrm{S}}$ at the source nodes, the optimal power allocation vector $\mathbf{p}_{\mathrm{R}}^{\mathrm{o}}$ at the relay will be first derived. Then, taking advantage of the symmetry of the received SNR at $\mathbf{D}$, the optimal power allocation vector $\mathbf{p}_{\mathrm{S}}^{\mathrm{o}}$ at the source nodes can be obtained if $\mathbf{p}_{\mathrm{R}}$ is given. Furthermore, we will propose an energy-efficient DIPA algorithm which is capable of achieving near-optimal EE performance for the entire relay system.

We present the following theorem to obtain $\mathbf{p}_{\mathrm{R}}^{\mathrm{o}}$.

Theorem 1. When the power allocation vector $\mathbf{p}_{S}$ is given at the source nodes, the SE of the kth node pair achieving the optimal $E E E_{b}^{o}$ is given by

$$
c_{k}^{o}=\left\{\log _{2}\left[\left(\sqrt{\delta_{R_{k}}+2}-\sqrt{\delta_{R_{k}}}\right) \sqrt{\frac{a_{k} p_{S_{k}}+1}{2}}\right]\right\}_{+},
$$

where

$$
\delta_{R_{k}}=\frac{\varsigma_{R} a_{k} p_{S_{k}} \ln 2}{\eta_{R} b_{k}\left(E_{b}^{o}-p_{C 1}\right)} .
$$

Proof. When $\mathbf{p}_{\mathrm{S}}$ is given in the feasible set, $p_{\mathrm{R}_{k}}$ can be given by plugging (4) into (6) as follows:

$$
p_{\mathrm{R}_{k}}\left(c_{k}\right)=\frac{\left(a_{k} p_{\mathrm{S}_{k}}+1\right) / b_{k}}{\left(\left(a_{k} p_{\mathrm{S}_{k}} /\left(2^{2 c_{k}}-1\right)\right)-1\right)} .
$$

Substituting (18) into (13), $P_{\Sigma}$ becomes a function of $\mathbf{c}$. The first-order partial derivative of $P_{\Sigma}(\mathbf{c})$ can be shown as

$$
\frac{\partial P_{\Sigma}}{\partial c_{k}}=\frac{a_{k} \varsigma_{\mathrm{R}} 2^{2 c_{k}+1}}{b_{k} \eta_{\mathrm{R}} \log _{2} \mathrm{e}} \frac{a_{k}+p_{\mathrm{S}_{k}}^{-1}}{\left[a_{k}-\left(2^{2 c_{k}}-1\right) p_{\mathrm{S}_{k}}^{-1}\right]^{2}}+p_{\mathrm{C} 1} .
$$

Moreover, the second-order partial derivative of $P_{\Sigma}(\mathbf{c})$ can be written as

$$
\frac{\partial^{2} P_{\Sigma}}{\partial c_{k}^{2}}=\frac{2}{\log _{2} \mathrm{e}}\left[\frac{2^{2 c_{k}+1} p_{\mathrm{S}_{k}}^{-1}}{a_{k}-\left(2^{2 c_{k}}-1\right) p_{\mathrm{S}_{k}}^{-1}}+1\right] \frac{\partial P_{\Sigma}}{\partial c_{k}} .
$$

Thus, $P_{\Sigma}(\mathbf{c})$ is a quasiconvex function with respect to $\mathbf{c}$, since the Hessian matrix of $P_{\Sigma}(\mathbf{c})$ is positive semidefinite; namely, $\nabla^{2} P_{\Sigma}(\mathbf{c})=\operatorname{diag}\left\{\partial^{2} P_{\Sigma} / \partial c_{1}^{2}, \partial^{2} P_{\Sigma} / \partial c_{2}^{2}, \ldots, \partial^{2} P_{\Sigma} / \partial c_{K}^{2}\right\} \geq 0$. Besides, $C_{\Sigma}(\mathbf{c})$ is an affine function of $\mathbf{c}$. Therefore, the lowerlevel set $\left\{\mathbf{c} \mid E_{\mathrm{b}}(\mathbf{c})=P_{\Sigma}(\mathbf{c}) / C_{\Sigma}(\mathbf{c}) \leq e_{\mathrm{b}}\right\}$ is a convex set, and the objective function of (15) is quasiconvex.

Letting $F\left(e_{\mathrm{b}}, \mathbf{c}\right)=P_{\Sigma}(\mathbf{c})-e_{\mathrm{b}} C_{\Sigma}(\mathbf{c}), F\left(e_{\mathrm{b}}, \mathbf{c}\right)$ is a convex function for a given $e_{\mathrm{b}} \geq 0$ and decreases with $e_{\mathrm{b}}$. According to [15], when $e_{\mathrm{b}}=E_{\mathrm{b}}^{\mathrm{o}}$, the objective function of the quasiconvex problem (15) can be transformed into

$$
F^{\mathrm{o}}\left(E_{\mathrm{b}}^{\mathrm{o}}, \mathbf{c}^{\mathrm{o}}\right)=\min _{\mathbf{c} \geq 0}\left\{P_{\Sigma}(\mathbf{c})-E_{\mathrm{b}}^{\mathrm{o}} C_{\Sigma}(\mathbf{c})\right\}
$$

which can be solved by the following stationary condition owing to its convexity:

$$
\frac{\partial F\left(E_{\mathrm{b}}^{\mathrm{o}}, \mathbf{c}\right)}{\partial c_{k}}=\frac{\partial P_{\Sigma}(\mathbf{c})}{\partial c_{k}}-E_{\mathrm{b}}^{\mathrm{o}}=0 .
$$

Through simplification, (22) can be rewritten as

$$
2^{2 c_{k}}+\sqrt{2\left(a_{k} p_{\mathrm{S}_{k}}+1\right) \delta_{\mathrm{R}_{k}}} 2^{c_{k}}-\left(a_{k} p_{\mathrm{S}_{k}}+1\right)=0 .
$$

Finally, solving the quadratic equation and saving the nonnegative root give rise to

$$
2^{c_{k}}=\left(\sqrt{\delta_{\mathrm{R}_{k}}+2}-\sqrt{\delta_{\mathrm{R}_{k}}}\right) \sqrt{\frac{a_{k} p_{\mathrm{S}_{k}}+1}{2}} .
$$

By applying the logarithmic operation to both sides of (24) and considering the nonnegativity of SE, (16) in the theorem is thus proved.

Substituting (16) into (18) leads to the optimal power allocation $p_{\mathrm{R}_{k}}^{\mathrm{o}}=\left[p_{\mathrm{R}_{k}}\left(c_{k}^{\mathrm{o}}\right)\right]_{+}=\left[p_{\mathrm{R}_{k}}\left(E_{\mathrm{b}}^{\mathrm{o}}, p_{\mathrm{S}_{k}}\right)\right]_{+}$for the $k$ th node pair at R. Since both $p_{\mathrm{R}_{k}}\left(c_{k}^{\mathrm{o}}\right)$ and $c_{k}^{\mathrm{o}}\left(E_{\mathrm{b}}^{\mathrm{o}}\right)$ are monotonically increasing functions, the composite function $p_{\mathrm{R}_{k}}^{\mathrm{o}}\left(E_{\mathrm{b}}^{\mathrm{o}}\right)$ is also a monotonically increasing one. Therefore, there exists an upper limit $E_{\mathrm{b}}^{\mathrm{U}}$ of the EE when considering the constraint $\mathbf{1}^{\mathrm{T}} \mathbf{p}_{\mathrm{R}} \leq P_{\mathrm{R}, \max }$. 
Step 1. (Initialization): $p_{S_{k}}=P_{S_{k}, \max }(k=1,2, \ldots, K), E_{b}^{U}=\infty, E_{b}^{L}=E_{b}^{L}\left(\mathbf{p}_{S}\right)$ in (28), and $\varepsilon>0$.

Step 2. (Update for relay node): $X=S$ and $Y=R$.

Sub-step 1: Executing the Sub-Algorithm.

Sub-step 2: $p_{R_{k}}^{o}=p_{R_{k}}\left(p_{S_{k}}, c_{k}^{o}\right), E_{b}^{U}=E_{b}^{o}\left(\mathbf{p}_{S}\right)$ and $E_{b}^{L}=E_{b}^{L}\left(\mathbf{p}_{R}^{o}\right)$.

Step 3. (Update for source nodes): $X=R$ and $Y=S$.

Sub-step 1: Executing the Sub-Algorithm.

Sub-step 2: $p_{S_{k}}^{o}=p_{S_{k}}\left(p_{R_{k}}, c_{k}^{o}\right), E_{b}^{U}=E_{b}^{o}\left(\mathbf{p}_{R}\right)$ and $E_{b}^{L}=E_{b}\left(\mathbf{p}_{S}^{o}\right)$.

Step 4. (Judgment): if $E_{b}^{o}\left(\mathbf{p}_{S}\right)-E_{b}^{o}\left(\mathbf{p}_{R}\right) \leq \varepsilon$, stop; else go back to Step 2.

Sub-Algorithm: Bi-section algorithm

Step 1. Let $u=1 / E_{b}^{U}$ and $v=1 / E_{b}^{L}$.

Step 2. Compute $\kappa=(u+v) / 2, e_{b}=1 / \kappa$ and $c_{k}^{o}\left(e_{b}, \mathbf{p}_{X}\right)$.

Step 3. Compute $f=F\left(e_{b}, \mathbf{c}^{o}\left(e_{b}, \mathbf{p}_{X}\right)\right)$. If $f>0$ and the constraint $T_{Y}$ is valid, then $u=\kappa$; Else $v=\kappa$.

Step 4. If $v-u<\varepsilon, E_{b}^{o}\left(\mathbf{p}_{X}\right)=e_{b}^{o}$; Else go back to Step 2 .

Algorithm 1: Energy-efficient dual-iteration power allocation.

According to the definition of function $F\left(e_{\mathrm{b}}, \mathbf{c}\right)$, the following relationships hold [16]:

$$
F\left(e_{\mathrm{b}}, \mathbf{c}\right) \begin{cases}>0, & e_{\mathrm{b}}<E_{\mathrm{b}}^{\mathrm{o}} \\ =0, & e_{\mathrm{b}}=E_{\mathrm{b}}^{\mathrm{o}} \\ <0, & e_{\mathrm{b}}>E_{\mathrm{b}}^{\mathrm{o}}\end{cases}
$$

Then, given $\mathbf{p}_{S}$ at $\mathbf{S}$ and considering the constraint $T_{R}$, the optimal $\mathbf{p}_{\mathrm{R}}^{\mathrm{o}}$ at $\mathrm{R}$ can be achieved by the bisection algorithm, which will be described in the subalgorithm of Algorithm 1 .

Similarly, given the power allocation $\mathbf{p}_{\mathrm{R}}$ at $\mathrm{R}$ and taking advantage of the symmetry of the received SNR at $\mathrm{D}_{k}$, the optimal SE of the $k$ th node pair can be shown as

$$
c_{k}^{\mathrm{o}}=\left\{\log _{2}\left[\left(\sqrt{\delta_{\mathrm{S}_{k}}+2}-\sqrt{\delta_{\mathrm{S}_{k}}}\right) \sqrt{\frac{b_{k} p_{\mathrm{R}_{k}}+1}{2}}\right]\right\}_{+},
$$

where

$$
\delta_{\mathrm{S}_{k}}=\frac{b_{k} \varsigma_{\mathrm{S}_{k}} p_{\mathrm{R}_{k}} \ln 2}{a_{k} \eta_{\mathrm{S}_{k}}\left(E_{\mathrm{b}}^{\mathrm{o}}-p_{\mathrm{C} 1}\right)} .
$$

Furthermore, considering the constraint $\mathrm{T}_{S}$, one can obtain $p_{\mathrm{S}_{k}}^{\mathrm{o}}=\left[p_{\mathrm{S}_{k}}\left(c_{k}^{\mathrm{o}}\right)\right]_{0}^{P_{\mathrm{S}_{k}, \max }}=\left[p_{\mathrm{S}_{k}}\left(E_{\mathrm{b}}^{\mathrm{o}}, p_{\mathrm{R}_{k}}\right)\right]_{0}^{P_{\mathrm{S}_{k}, \max }}$. Therefore, the optimal $\mathbf{p}_{\mathrm{S}}^{\mathrm{o}}$ achieving the optimal EE at $\mathbf{S}$ is also attainable by the bisection algorithm when $\mathbf{p}_{\mathrm{R}}$ at $\mathrm{R}$ is given.

Considering the power allocation for both phases, we develop an energy-efficient DIPA algorithm for multiple node pairs at both $\mathbf{S}$ and $\mathrm{R}$ as detailed in Algorithm 1 .

In the DIPA algorithm, given the transmission power $\mathbf{p}_{\mathrm{X}}$ at $\mathrm{X}(=\mathbf{S}$ or $\mathrm{R})$, ignoring the transmission power $\mathbf{p}_{\mathrm{Y}}$ at $\mathrm{Y}(=$ $\mathrm{R}$ or $\mathbf{S})$ and replacing the SE $\mathbf{c}$ with its upper bound $\mathbf{c}^{\mathrm{U}}\left(\mathbf{p}_{\mathrm{X}}\right)=$ $\left[c_{1}^{\mathrm{U}}, c_{2}^{\mathrm{U}}, \ldots, c_{\mathrm{K}}^{\mathrm{U}}\right]^{\mathrm{T}}$, a lower-bounded EE $E_{\mathrm{b}}^{\mathrm{L}}$ can be obtained as follows:

$$
E_{\mathrm{b}}^{\mathrm{L}}\left(\mathbf{p}_{\mathrm{X}}\right)=\frac{P_{\text {other }}\left(\mathbf{p}_{\mathrm{X}}\right)}{\mathbf{1}^{\mathrm{T}} \mathbf{c}^{\mathrm{U}}\left(\mathbf{p}_{\mathrm{X}}\right)},
$$

where

$$
P_{\text {other }}\left(\mathbf{p}_{\mathrm{X}}\right)=P_{\Sigma}-\frac{\varsigma_{\mathrm{Y}}}{\eta_{\mathrm{Y}}} \mathbf{1}^{\mathrm{T}} \mathbf{p}_{\mathrm{Y}}
$$

and a SE upper bound $c_{k}^{U}$ for the $k$ th node pair is

$$
c_{k}^{\mathrm{U}}\left(p_{\mathrm{X}_{k}}\right)=\frac{1}{2} \log _{2}\left(1+p_{\mathrm{X}_{k}} \gamma_{k}\right)
$$

where $\gamma_{k}=a_{k}$ if $\mathrm{X}=\mathbf{S}$; else $\gamma_{k}=b_{k}$.

4.2. DIPA Based on Statistic CSI. As Algorithm 1 is dependent on the fast fading channels, namely, the instantaneous CSI, the different symbols on different subcarriers from the same source node should be calculated separately. Therefore, the complexity is overwhelming for practical systems. Thanks to the channel harden phenomenon of massive MIMO, the norm of each channel link can be approximated by the largescale fading multiplied by the number of antennas [5-7]. Then, we can replace the instantaneous $\left(a_{k}, b_{k}\right)$ in (5) with the statistic $\left(\bar{a}_{k}, \bar{b}_{k}\right)$ in (9) in the DIAP algorithm. In other words, the instantaneous SE expression in (6) can be replaced with the ergodic lower bound in (7) during the derivations of Theorem 1. Thus, the proposed algorithm can be implemented efficiently owing to nearly the same large-scale fading for different subcarriers of each link. Moreover, the method of DIPA based on both the statistic CSI and instantaneous CSI can be easily extended to other precoders and detectors. And the stochastic analysis of relay with massive antennas under Markov channel is our future interest [17].

\section{Simulation and Discussions}

This section evaluates the EE and SE of the proposed energyefficient DIPA algorithms compared with the EPA algorithm. The results of DIPA based on the instantaneous CSI and statistic CSI are denoted by "IC" and "SC", respectively, in Figures 3-6. For the purpose of fair comparison, the total radiated power of the source nodes or relay node used in the EPA algorithm is made the same as the actual power consumed by the proposed DIPA algorithm. In order to simplify the simulation, without loss of generality, it is assumed that $\eta_{\mathrm{S}_{k}}=\eta_{\mathrm{R}}=\eta, \varsigma_{\mathrm{S}_{k}}=\varsigma_{\mathrm{R}}=\varsigma, \sigma_{\mathrm{sh}, \mathrm{S}_{k} \mathrm{R}}^{2}=\sigma_{\mathrm{sh}, \mathrm{RD}_{k}}^{2}=$ $\sigma_{\mathrm{sh}}^{2}, \sigma_{\mathrm{R}}^{2}=\sigma_{\mathrm{D}_{k}}^{2}=\sigma^{2}$, and $P_{\mathrm{S}_{k}, \max }=P_{\mathrm{S}, \max }$. Similar to $[13,14]$, 
TABLE 1: Simulation parameters for the relay system.

\begin{tabular}{lc}
\hline Parameters & Values \\
\hline$\phi$ & 1 \\
$\eta$ & 0.35 \\
$\varsigma$ & 1.2705 \\
$\alpha$ & 3.7 \\
$\sigma_{\mathrm{sh}}$ & $8 \mathrm{~dB}$ \\
$r_{0}$ & $35 \mathrm{~m}$ \\
$\sigma^{2}$ & $-124 \mathrm{dBm} / \mathrm{Hz}$ \\
$\varepsilon$ & $1 E-8$ \\
$\Delta f$ & $15 \mathrm{kHz}$ \\
$p_{\mathrm{DC} 1}=2 p_{\mathrm{SC} 1}$ & $0.2 \mathrm{~mW} / \Delta f$ \\
$p_{\mathrm{RC} 1}$ & $0.5 \mu \mathrm{W} / \Delta f$ \\
$p_{\mathrm{RC} 2}=2 p_{\mathrm{SC} 2}=2 p_{\mathrm{DC} 2}$ & $0.8 \mathrm{~mW} / \Delta f$ \\
$p_{\mathrm{RC} 3}=2 p_{\mathrm{SC} 3}=2 p_{\mathrm{DC} 3}$ & $2 \mathrm{~W}$ \\
$p_{\mathrm{RC} 4}$ & $10 \mathrm{~W}$ \\
$P_{S, \max }$ & $1 \mu \mathrm{W} / \mathrm{Hz}$ \\
$P_{R, \max }$ & $1 \mathrm{~mW} / \mathrm{Hz}$ \\
\hline
\end{tabular}

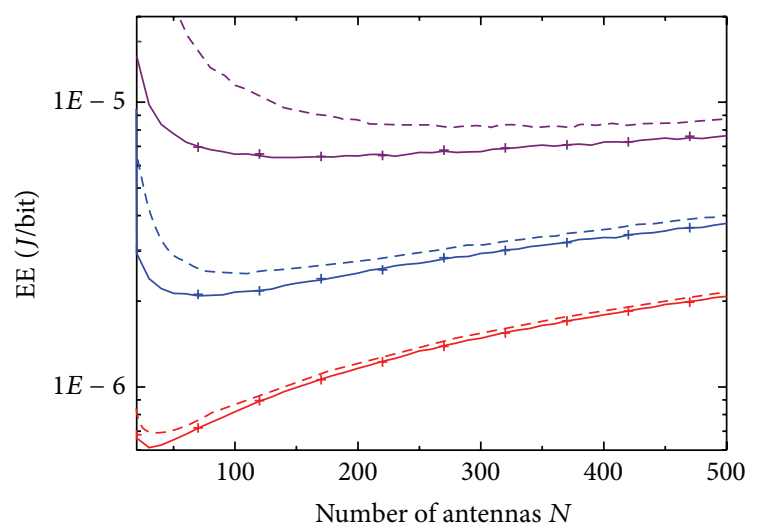

$$
\begin{aligned}
& K=10 \\
& \text { IC SC EPA } R_{0} \\
& \text { - + - } 0.2 \mathrm{~km} \\
& +---0.5 \mathrm{~km} \\
& ++--1 \mathrm{~km}
\end{aligned}
$$

FIgURE 3: EE versus the number of antennas $N$ at the relay.

the main parameters used in our simulations are listed in Table 1. During each instance of simulation, the source nodes or destination nodes are uniformly distributed in a given area with $\theta=60^{\circ}, r_{0}=35 \mathrm{~m}$, and different $R_{0}$, as shown in Figure 1.

Figure 3 plots the average EE versus the number of antennas at the relay node with various radii $R_{0}$, when the number of node pairs $K$ is 10 . Figure 4 shows the average EE versus the number of node pairs with different numbers of antennas $N$ at the relay node when the radius $R_{0}$ is $0.5 \mathrm{~km}$. Both Figures 3 and 4 indicate that the proposed DIPA algorithms can improve the EE compared with the EPA algorithm, which validates the proposed DIPA algorithms. The results of DIPA based on the statistic CSI agree with the results of DIPA based on the instantaneous CSI. Thus, the DIPA based on

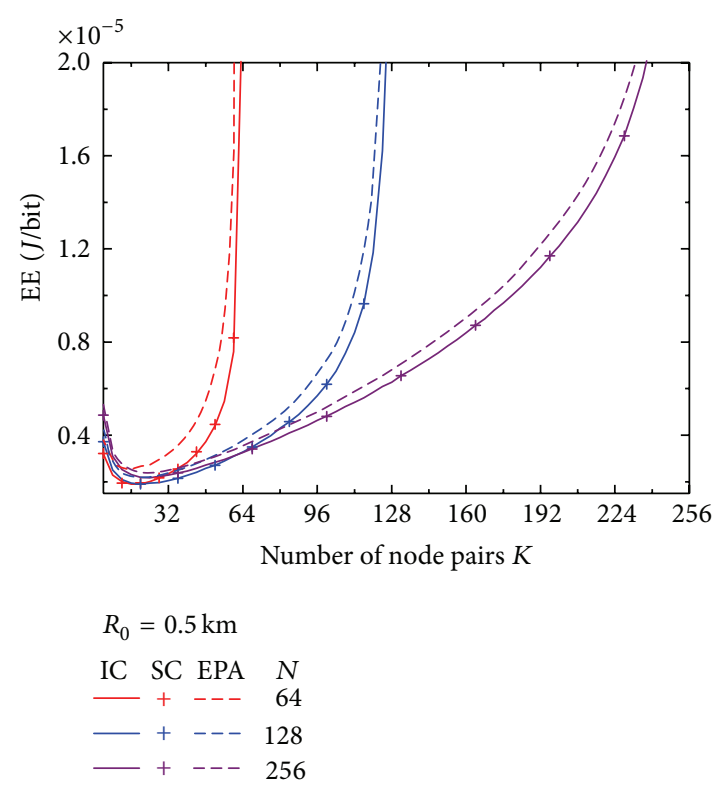

FIGURE 4: EE versus the number of node pairs $K$.

the statistic CSI is more advisable for practical communication systems. Moreover, the EE first decreases and then increases with respect to both the number of antennas of the relay node and the number of multiple node pairs, since the rate of SE increase is first faster and then slower than the rate of power increase. Therefore, deploying a reasonable number of antennas at the relay node and multiplexing a rational number of node pairs can enhance the system EE. Furthermore, Figure 3 indicates that shrinking the radius of the serving area can enhance the EE, since less power is consumed to compensate the path loss.

The corresponding SEs to both Figures 3 and 4 are given in Figures 5 and 6, respectively. Because the algorithms consume the same amount of power, the DIPA algorithms perform better than the EPA algorithm in terms of SE according to the definition of EE. The DIPA based on the statistic CSI performs the same as the DIPA based on the instantaneous CSI. Both figures demonstrate that SE can be improved by deploying more antennas at the relay node. Multiplexing a rational number of node pairs and shrinking the radius of the severing area can improve system SE, as can be observed in Figures 6 and 5, respectively.

\section{Conclusion}

This paper studied energy-efficient power allocation at both source nodes and relay node for a two-phase AF relay system, where the relay is equipped with massive antennas and employs backward and forward ZF filters. Through decomposing the formulated EE optimization problem into two phases, two DIPA algorithms are proposed based on the instantaneous CSI and statistic CSI, respectively. Simulation results demonstrate the effectiveness of the proposed algorithms compared with the EPA algorithm. The DIPA algorithm based on the statistic CSI is more advisable compared 


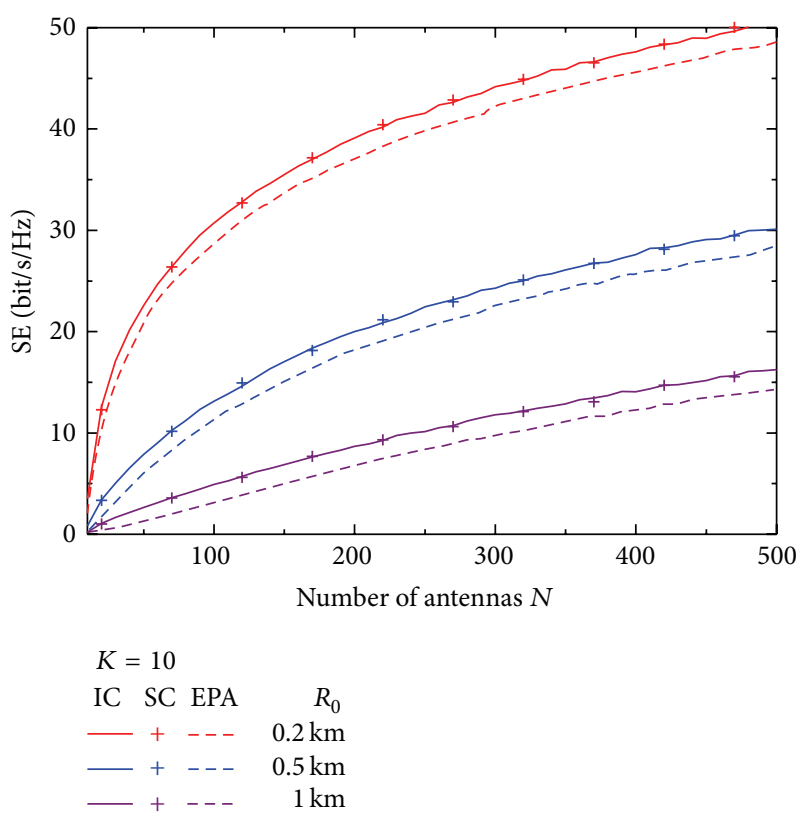

FIGURE 5: SE versus the number of antennas $N$ at the relay.

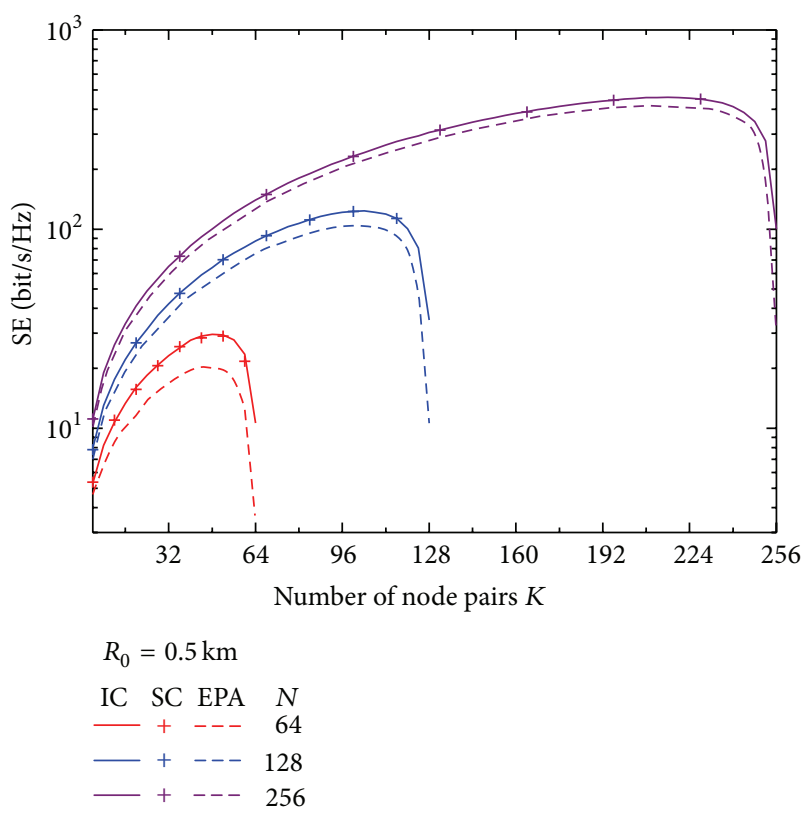

FIgURE 6: SE versus the number of node pairs $K$.

with that based on the instantaneous CSI for practical systems in consideration of implementation complexity. Moreover, deploying a rational number of antennas at the relay and multiplexing a reasonable number of node pairs can improve the system performance. Shrinking the radius of the severing area is also an important measure for better SE and EE.

\section{Conflict of Interests}

The authors declare that there is no conflict of interests regarding the publication of this paper.

\section{Acknowledgments}

This work was supported by the National 863 Program of China (2014AA01A705), National Key Technology R\&D Program of China (2012ZX03004005), China Natural Science Funding (61271183), and Research Fund for the Doctoral Program of Higher Education (20130005120003).

\section{References}

[1] Z. Hasan, H. Boostanimehr, and V. K. Bhargava, "Green cellular networks: a survey, some research issues and challenges," IEEE Communications Surveys and Tutorials, vol. 13, no. 4, pp. 524540, 2011.

[2] A. Fehske, G. Fettweis, J. Malmodin, and G. Biczok, "The global footprint of mobile communications: the ecological and economic perspective," IEEE Communications Magazine, vol. 49, no. 8, pp. 55-62, 2011.

[3] T. L. Marzetta, "Noncooperative cellular wireless with unlimited numbers of base station antennas," IEEE Transactions on Wireless Communications, vol. 9, no. 11, pp. 3590-3600, 2010.

[4] H. Q. Ngo, E. G. Larsson, and T. L. Marzetta, "Energy and spectral efficiency of very large multiuser MIMO systems," IEEE Transactions on Communications, vol. 61, no. 4, pp. 1436-1449, 2013.

[5] F. Rusek, D. Persson, K. L. Buon et al., "Scaling up MIMO: opportunities and channenges with very large arrays," IEEE Signal Processing Magazine, vol. 30, no. 1, pp. 40-60, 2013.

[6] E. G. Larsson, F. Tufvesson, O. Edfors, and T. L. Marzetta, "Massive MIMO for next generation wireless systems," IEEE Communications Magazine, vol. 52, no. 2, pp. 186-195, 2014.

[7] B. M. Hochwald, T. L. Marzetta, and V. Tarokh, "Multipleantenna channel hardening and its implications for rate feedback and scheduling," IEEE Transactions on Information Theory, vol. 50, no. 9, pp. 1893-1909, 2004.

[8] J. Hoydis, S. ten Brink, and M. Debbah, "Massive MIMO in the UL/DL of cellular networks: how many antennas do we need?" IEEE Journal on Selected Areas in Communications, vol. 31, no. 2, pp. 160-171, 2013.

[9] H. Yang and T. L. Marzetta, "Performance of conjugate and zeroforcing beamforming in large-scale antenna system," IEEE Journal on Selected Areas in Communications, vol. 31, no. 2, pp. 172-179, 2013.

[10] A. S. Himal, Q. N. Hien, Q. D. Trung, Y. Chau, and G. L. Erik, "Multipair amplify-and-forward relaying with very large antenna arrays," in Proceedings of the IEEE International Conference on Communications (ICC '13), pp. 3228-3233, Budapest, Hungary, June 2013.

[11] R. Couillet and M. Debbah, Random Matrix Methods for Wireless Communications, Cambridge University Press, New York, NY, USA, 2011.

[12] L. Zhao, K. Zheng, H. Long, and H. Zhao, "Performance analysis for downlink massive MIMO system with ZF precoding," Transactions on Emerging Telecommunications Technologies, 2013.

[13] H. S. Kim and B. Daneshrad, "Energy-constrained link adaptation for MIMO OFDM wireless communication systems," IEEE Transactions on Wireless Communications, vol. 9, no. 9, pp. 2820-2832, 2010.

[14] Z. Xu, C. Yang, G. Y. Li, S. Zhang, Y. Chen, and S. Xu, "Energyefficient configuration of spatial and frequency resources in 
MIMOOFDM sytems," IEEE Transactions on Communications, vol. 61, no. 2, pp. 564-575, 2013.

[15] C. Isheden, Z. Chong, E. Jorswieck, and G. Fettweis, "Framework for link-level energy efficiency optimization with informed transmitter," IEEE Transactions on Wireless Communications, vol. 11, no. 8, pp. 2946-2957, 2012.

[16] S. Boyd and L. Vandenberghe, Convex Optimization, Cambridge University Press, New York, NY, USA, 2004.

[17] K. Zheng, F. Liu, L. Lei, C. Lin, and Y. Jiang, "Stochastic performance analysis of a wireless finite-state markov channel," IEEE Transactions on Wireless Communications, vol. 12, no. 2, pp. 782793, 2013 

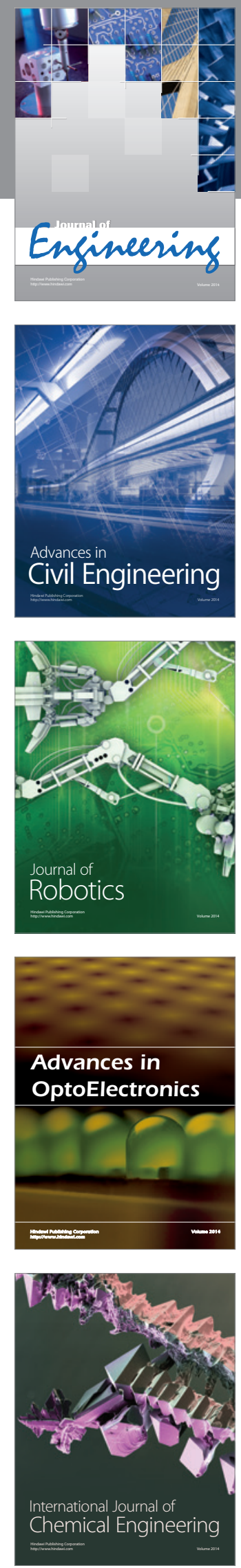

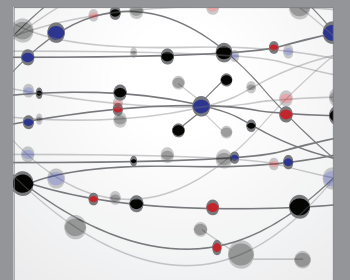

The Scientific World Journal
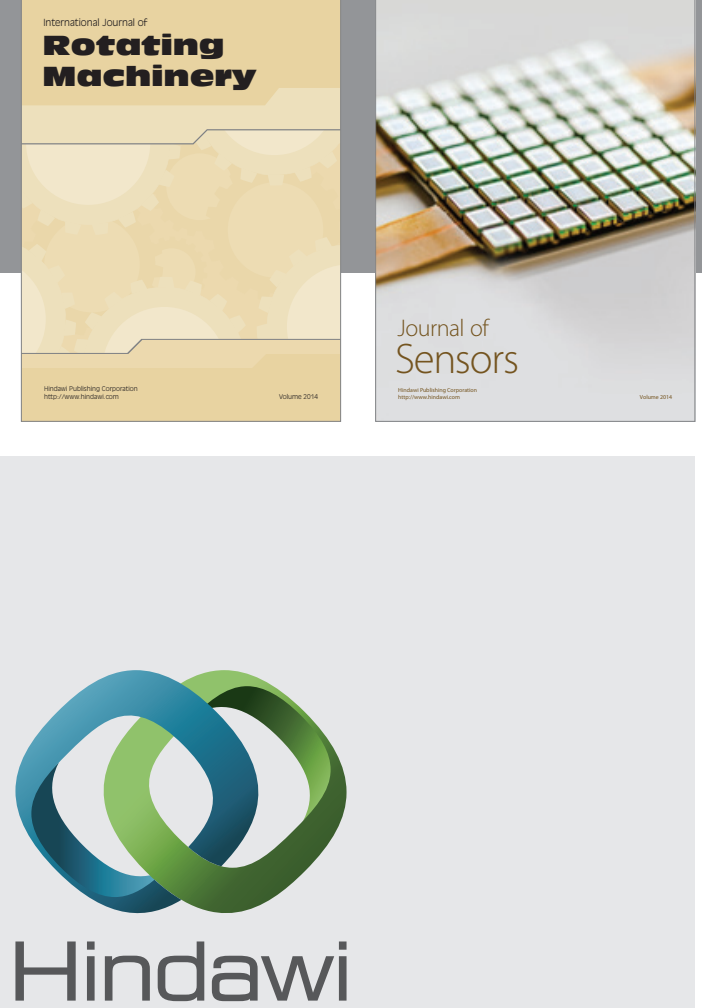

Submit your manuscripts at http://www.hindawi.com
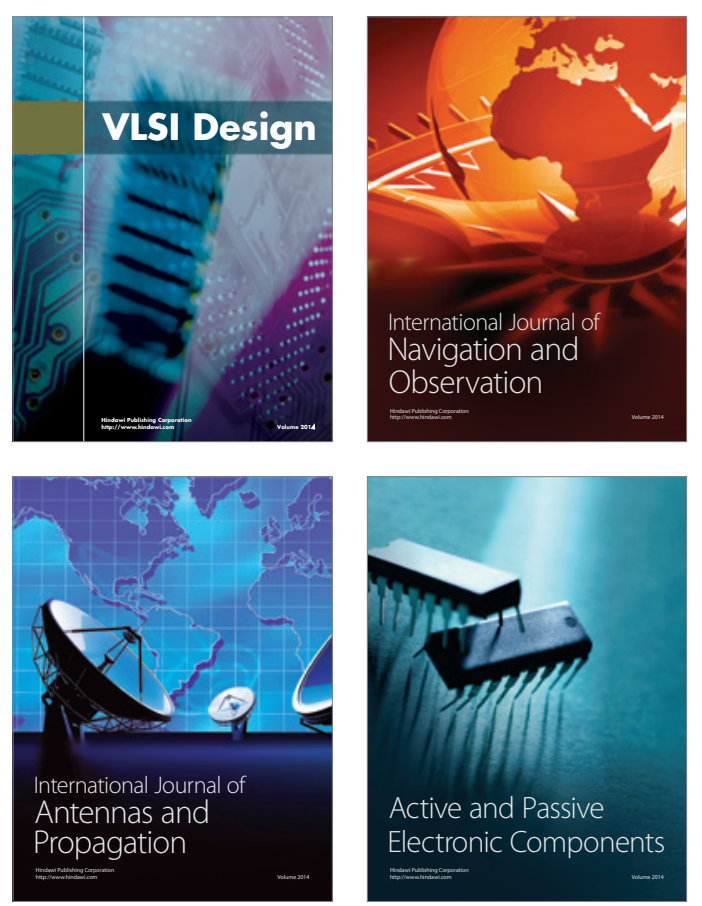
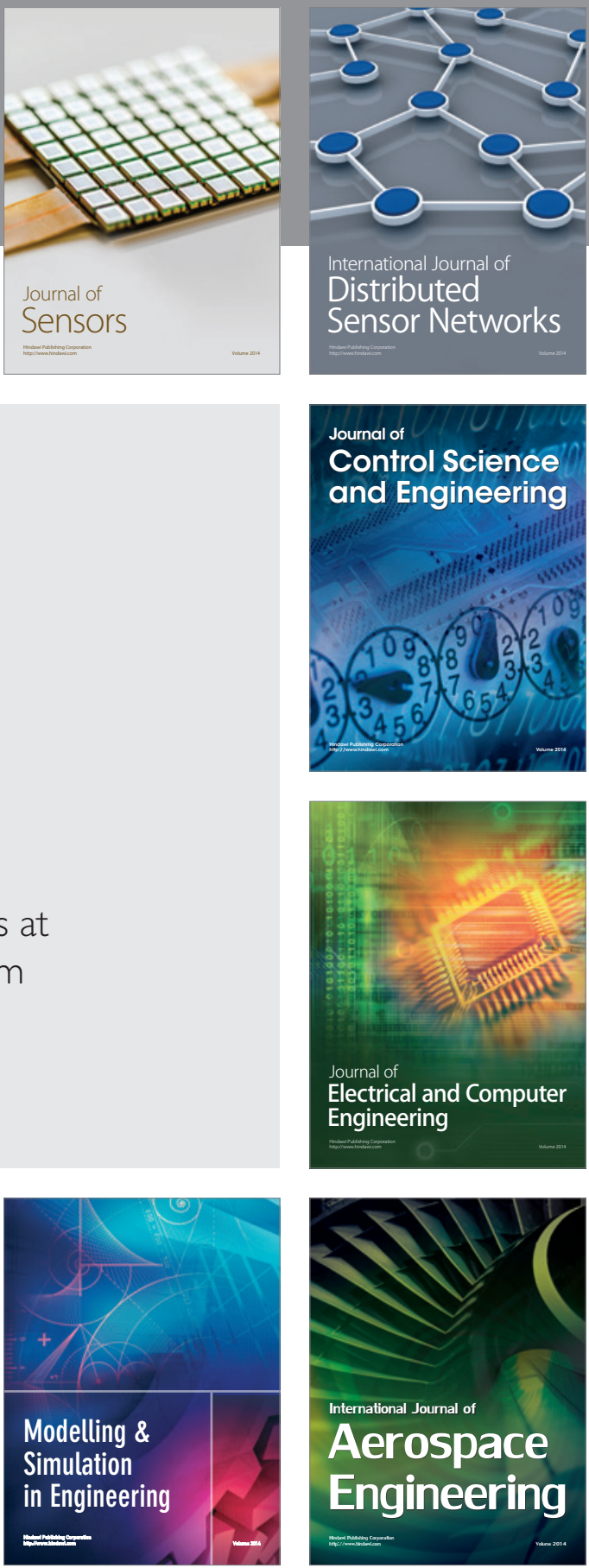

Journal of

Control Science

and Engineering
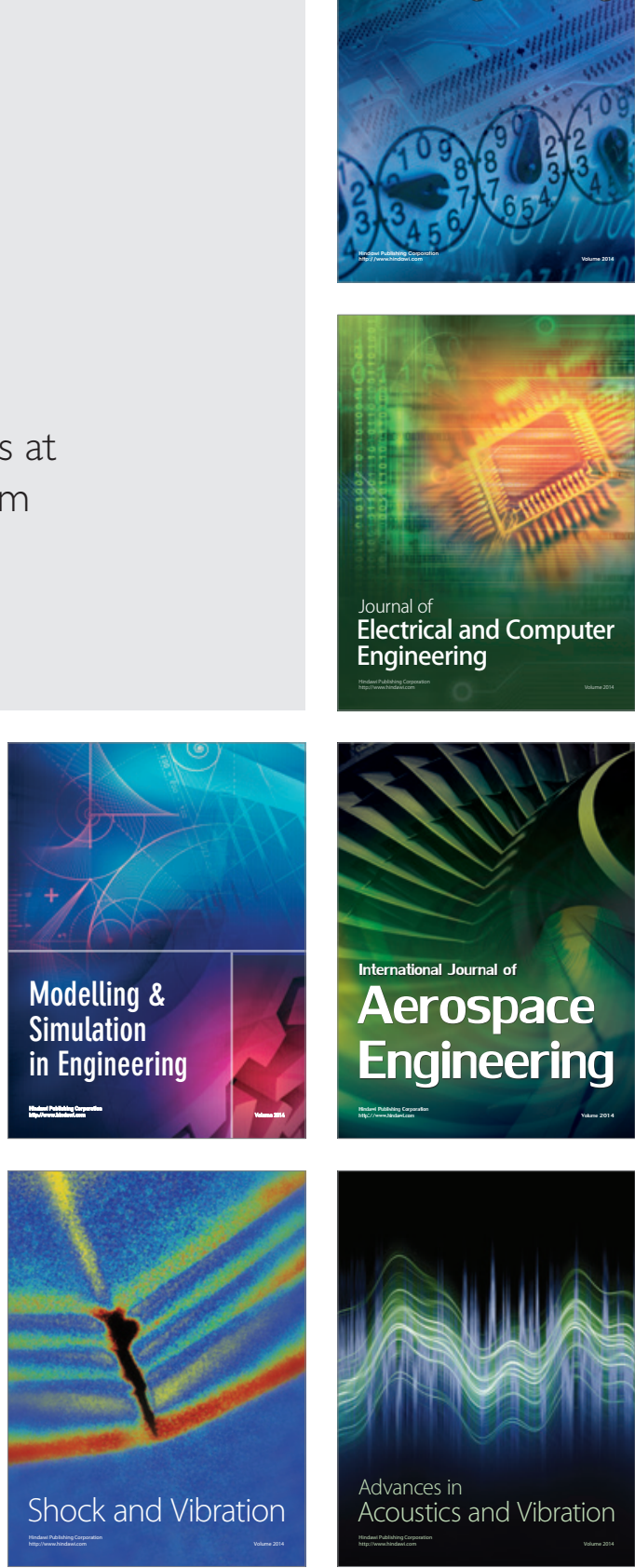